\title{
Effect of eicosapentaenoic acid and pitavastatin on electrophysiology and anticoagulant gene expression in mice with rapid atrial pacing
}

\author{
MAOQING TONG $^{1 *}$, JIEWEN WANG $^{2 *}$, YUNXIN JI $^{3 *}$, XIAOMIN CHEN $^{4}$, \\ JIERU WANG ${ }^{5}$, SHUANGSHUANG WANG ${ }^{4}$, LIEMIN RUAN ${ }^{3}$, HANBIN CUI $^{4}$, \\ YING ZHOU $^{1}$, QINGYU ZHANG ${ }^{3}$ and EIICHI WATANABE ${ }^{6}$
}

\begin{abstract}
${ }^{1}$ Department of Cardiology, Key Laboratory of Molecular Medicine, The Affiliated Ningbo First Hospital, School of Medicine, Ningbo University, Ningbo, Zhejiang 315010; ${ }^{2}$ Department of Gynecology and Obstetrics, The Second Affiliated Hospital of Bengbu Medical College, Bengbu, Anhui 233040; Departments of ${ }^{3}$ Psychosomatic Medicine, ${ }^{4}$ Cardiology and ${ }^{5}$ International Health, The Affiliated Ningbo First Hospital, School of Medicine, Ningbo University, Ningbo, Zhejiang 315010, P.R. China; ${ }^{6}$ Department of Cardiology, Fujita Health University School of Medicine, Toyoake 470-1192, Japan
\end{abstract}

Received November 8, 2016; Accepted April 21, 2017

DOI: $10.3892 / \mathrm{etm} .2017 .4741$

\begin{abstract}
Atrial remodeling is considered to be any persistent change in atrial structure or function, and is responsible for the development and perpetuation of atrial fibrillation (AF). Oxidative stress and intracellular $\mathrm{pH}$ regulation may also be linked to AF; however it remains unclear whether eicosapentaenoic acid (EPA) or statins have beneficial therapeutic effects. The aim of the present study was to investigate the effects of EPA and pitavastatin on the electrophysiology of and gene expressions in mice with rapidly-paced atria. Mice were treated with EPA $(10 \mathrm{mg} / \mathrm{g} /$ day $)$ or pitavastatin $(30 \mathrm{ng} / \mathrm{g} /$ day $)$ for 6 weeks, following which AF was simulated by 8 -h atrial pacing at $1,800 \mathrm{bpm}$. The atrial electrophysiological properties and the expression of cardiac genes, potassium voltage-gated channel subfamily A member 5 (Kcna5), Kcn subfamily $\mathrm{D}$ member 2 (Kcnd2), Kv channel-interacting protein 2 (KChIP2), solute carrier family 9 member A1, thrombomodulin (TM) and tissue factor pathway inhibitor (TFPI) were examined using reverse transcription-quantitative polymerase chain reaction. In control mice, significant atrial electrical remodeling was observed $(\mathrm{P}<0.05)$; however, treatment with either EPA or pitavastatin ameliorated these electrophysiological changes (P>0.05). mRNA levels of Kcnd2, KChIP2 and Kcna5 were
\end{abstract}

Correspondence to: Dr Maoqing Tong, Department of Cardiology, Key Laboratory of Molecular Medicine, The Affiliated Ningbo First Hospital, School of Medicine, Ningbo University, 59 Liuting Road, Ningbo, Zhejiang 315010, P.R. China

E-mail: ganbarusy@163.com

*Contributed equally

Key words: atrial fibrillation, remodeling, cardiac ion channel, oxidative stress significantly upregulated in control mice $(\mathrm{P}<0.05)$, whereas treatment with EPA or pitavastatin attenuated this upregulation $(\mathrm{P}>0.05)$. Administration of pitavastatin significantly reduced the downregulation of both TFPI and TM $(\mathrm{P}<0.05)$. EPA treatment attenuated the TFPI downregulation compared with control mice $(\mathrm{P}>0.05)$, however no significant effect on TM expression was observed. In addition, both EPA $(\mathrm{P}>0.05)$ and pitavastatin $(\mathrm{P}<0.05)$ suppressed the overexpression of endothelial nitric oxide synthase. This was also exhibited in Ras-related C3 botulinum toxin substrate 1 genes $(\mathrm{P}<0.01$ for both treatments). In conclusion, the results of the present study suggested that EPA and pitavastatin are able to prevent atrial electrical remodeling, thrombotic states and oxidative stress in rapidly-paced murine atria.

\section{Introduction}

Atrial fibrillation (AF) is the most common sustained cardiac arrhythmia and is becoming progressively more prevalent in the aging population (1). AF is associated with an increased risk of thromboembolism and mortality (2). The development and perpetuation of AF is dependent on the electrophysiological and structural substrate remodeling of the atria (3-5).

Electrical remodeling represents a series of alterations in ion channel expression and/or function, which in turn shorten the atrial refractory period and lead to a decrease in conduction velocity (3). A previous study demonstrated an increased expression of the transient outward $\mathrm{K}^{+}$current $\left(\mathrm{I}_{\mathrm{to}}\right)$ and ultra-rapid delayed rectifier $\mathrm{K}^{+}$current $\left(\mathrm{I}_{\mathrm{kur}}\right)$ in the atria of rats with rapid atrial pacing (6). In a canine experiment, activation of the $\mathrm{Na}^{+} / \mathrm{H}^{+}$exchanger was reported as a possible underlying mechanism of electrophysiological remodeling (7).

The role of oxidative stress in the pathophysiology of atrial remodeling has been widely researched (8). In particular, NADPH oxidase (NOX) has emerged as a potential enzymatic source for reactive oxygen species (ROS) production in AF. Ras-related C3 botulinum toxin substrate 1 (Rac1) is important 
for the membrane subunit assembly required to form an active NOX complex $(9,10)$. However, the relationship between Rac1 and cardiac ion channels remains to be elucidated.

Patients with AF have up to a 5-fold higher risk of suffering ischemic strokes (11). A better understanding of the etiologies of thromboembolic complications is required to improve patient care and treatment options. A study by Yamashita et al (12) demonstrated that thrombomodulin (TM) and tissue factor pathway inhibitor (TFPI) genes were downregulated in the atrial endocardium of a rat model of rapid atrial pacing, and this may induce a local coagulation imbalance on the internal surface of the atrial cavity. Increased understanding of atrial remodeling has introduced the possibility of upstream therapeutic approaches targeting the atrial substrates that initiate and perpetuate AF $(2,13)$. Although the exact mechanism has not yet been established, the anti-AF effect of statins and eicosapentaenoic acid (EPA) may be associated with an improvement in lipid metabolism and anti-inflammatory and antioxidant actions (14-20). Furthermore, previous experimental reports have indicated that these pharmacological agents reduce AF inducibility and conduction heterogeneity $(21,22)$. However, few studies have compared the effect of EPA and statins on the electrophysiological characteristics and the expression of ion channel genes related to electrical remodeling, intrinsic anticoagulant factor genes and endothelial nitric oxide synthase genes that may be associated with thromboembolic events complicated with AF. In the present study, the anti-AF effects of EPA and statins were investigated using a murine model of rapid atrial pacing.

\section{Materials and methods}

Preparation of the atrial tachycardia models and electrophysiological study. Animal research was reviewed and approved by the Ethics Committee of Ningbo University School of Medicine (Ningbo, China). A total of 60 male ddY mice (weight, 25 $\pm 0.5 \mathrm{~g}$; aged 3 weeks) were purchased from Chubu Kagaku Shizai Co., Ltd., (Nagoya, Japan). Mice were housed for 6 weeks in an animal facility at $22 \pm 1{ }^{\circ} \mathrm{C}$ under a 12-h light/dark cycle with $70 \%$ humidity and were provided with food and water ad libitum. Mice were divided randomly into three groups: Control, EPA treatment and pitavastatin treatment ( $n=20$ in each group). In the experimental groups, the mice were administered orally with EPA (10 mg/g/day; Ningbo Hangjing Co., Ltd., Ningbo, China) or pitavastatin(30 ng/g/day; NingboHangjingCo.,Ltd.)for6weeks. A murine model of atrial tachycardia was prepared using a previously described method with modifications (6). Briefly, mice were anesthetized subcutaneously with pentobarbital (50 mg/kg; Ningbo Hangjing Co., Ltd.) and ventilated with a small animal ventilator (SAR-830; CWE Inc., Ardmore, PA, USA). A quadripolar electrode catheter $(2.0 \mathrm{Fr})$ was introduced via the cervical vein for pacing and recording in anaesthetized mice (Fig. 1). Stimulation at a frequency of 1,800 bpm was performed with 2-msec rectangular pulses using a programmable stimulator and a constant current source (SEN 7203 and SS401J, respectively; Nihon Kohden, Tokyo, Japan). The intracardiac electrocardiogram (ECG) was recorded using a Biopac ECG $100 \mathrm{C}$ amplifier and sampled at a rate of 1,000 Hz with the Biopac MP150 data acquisition system (BIOPAC Systems,
Inc., Goleta, CA, USA). The effective refractory period (ERP) of the right atrium was measured before and after pacing at basic cycle lengths (BCLs) of 100 and $150 \mathrm{msec}$ (Fig. 2). The surface ECG was recorded using a DSI Dataquest A.R.T.2.3 system at a sampling rate of $1 \mathrm{kHz}$ (Data Sciences International, St. Paul, MN, USA) with a telemetry transmitter fixed on the mice as described previously (23). After $8 \mathrm{~h}$ of pacing, the left atrial appendages were harvested within $30 \mathrm{sec}$ and the tissues were immediately frozen in liquid nitrogen for subsequent RNA isolation.

$R N A$ preparation and reverse transcription-quantitative polymerase chain reaction ( $R T-q P C R)$. Mice were sacrificed by overdose with ketamine and xylazine $(125$ and $50 \mathrm{mg} / \mathrm{kg}$; Ningbo Hangjing Co., Ltd.) and death of mice were confirmed by cardiopulmonary arrest following ketamine/xylazine injection. Hearts were harvested immediately. Total RNA was extracted from the atrial samples using an RNeasyl Fibrous Tissue Mini kit (Qiagen AB, Sollentuna, Sweden), according to the manufacturer's instructions, reverse transcribed and amplified using real-time SYBR Green technology $\left(\mathrm{SYBR}^{\circledR}\right.$ Premix Ex TaqTM II kit; Takara Biotechnology Co., Ltd., Beijing, China), and analyzed using an ABI PRISM7900 (Applied Biosystems; Thermo Fisher Scientific, Inc., Waltham, MA, USA). The PCR data are reported as the number of transcripts per number of GAPDH. The primer sequences are presented in Table I and were designed based on the mouse sequences available in GenBank (https://www.ncbi.nlm.nih.gov/genbank/), including three $\mathrm{K}^{+}$channel genes: Potassium voltage-gated channel subfamily A member 5 (Kcna5), Kcn subfamily D member 2 (Kcnd2), Kv channel-interacting protein 2 (KChIP2), solute carrier family 9 member A1 (Slc9a1), two intrinsic anticoagulant factors (TM and TFPI) and the oxidative critical component of NOX (Rac1). RT-qPCR was performed under the following conditions: cDNA synthesis at $25^{\circ} \mathrm{C}$ for $10 \mathrm{~min}$ and then $37^{\circ} \mathrm{C}$ for $120 \mathrm{~min}$, and PCR amplification for 40 cycles at $95^{\circ} \mathrm{C}$ for $15 \mathrm{sec}$ and $60^{\circ} \mathrm{C}$ for $1 \mathrm{~min}$. PCR data were reported as the number of transcripts per number of GAPDH genes.

Statistical analysis. All values were expressed as the mean \pm standard error of the mean. Multiple-group statistical comparisons were obtained using one-way analysis of variance. Student's t-test with a Tukey-Kramer correction was used to evaluate the differences between individual means. SPSS 19.0 software was used (IBM Corp.- Armonk, NY, USA) and $\mathrm{P}<0.05$ was considered to indicate a statistically significant difference.

\section{Results}

Rapid atrial pacing model. The surface ECG and atrial electrograms obtained in the murine model of rapid atrial pacing are presented in Fig. 1. Each pacing signal had its corresponding ventricular complex, which indicated that the atrium could be captured by rapid pacing at 1,800 bpm. Fig. 2 demonstrates the method for measuring the ERP.

Effect of EPA treatment on atrial electrical remodeling. The ERP was measured before and after rapid atrial pacing at $1,800 \mathrm{bpm}$ for over $8 \mathrm{~h}$. In the control group, the ERP decreased significantly from $65.3 \pm 2.1$ to $54.3 \pm 1.5 \mathrm{msec}$ at a BCL of 


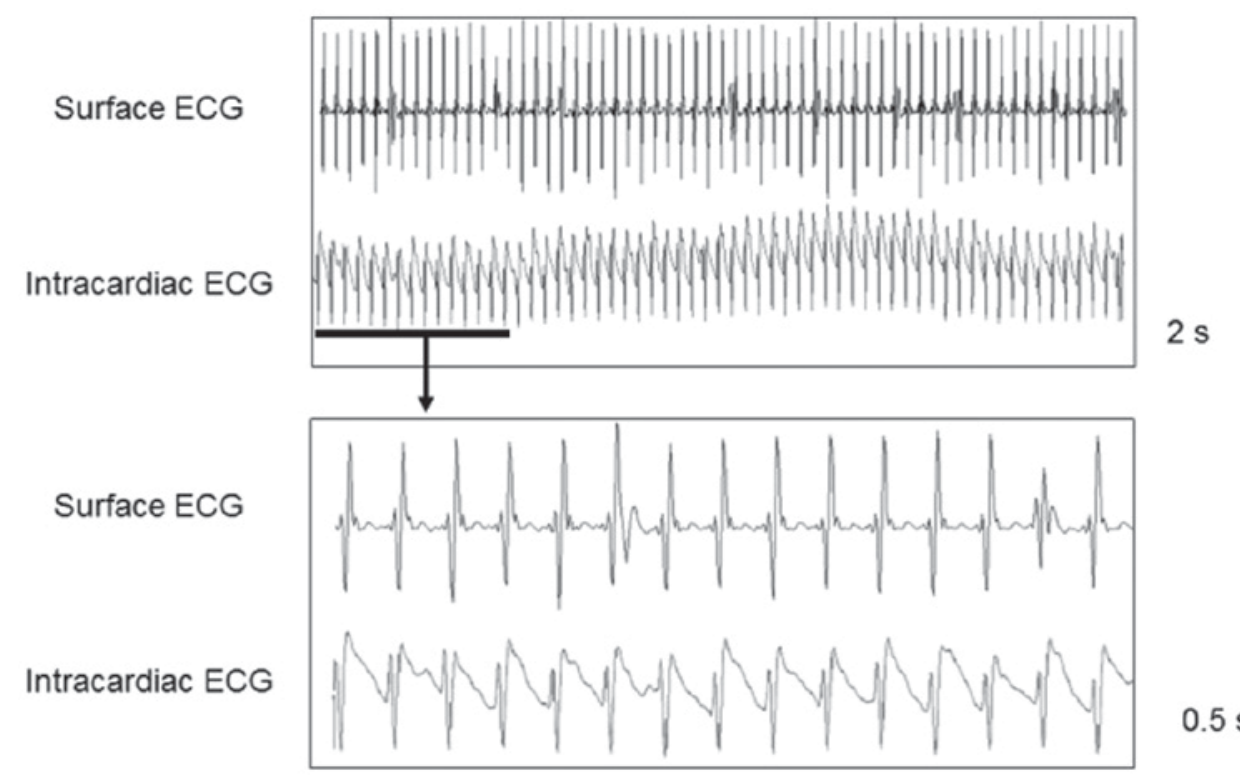

Figure 1. Murine model of rapid atrial pacing. The right external jugular vein was cannulated and a quadripolar electrode catheter was inserted into the right atrium for pacing and recording. The surface and intracardiac ECGs confirmed that the atrial fibrillation model was successfully established by pacing at $1,800 \mathrm{bpm}$. ECG, electrocardiogram.

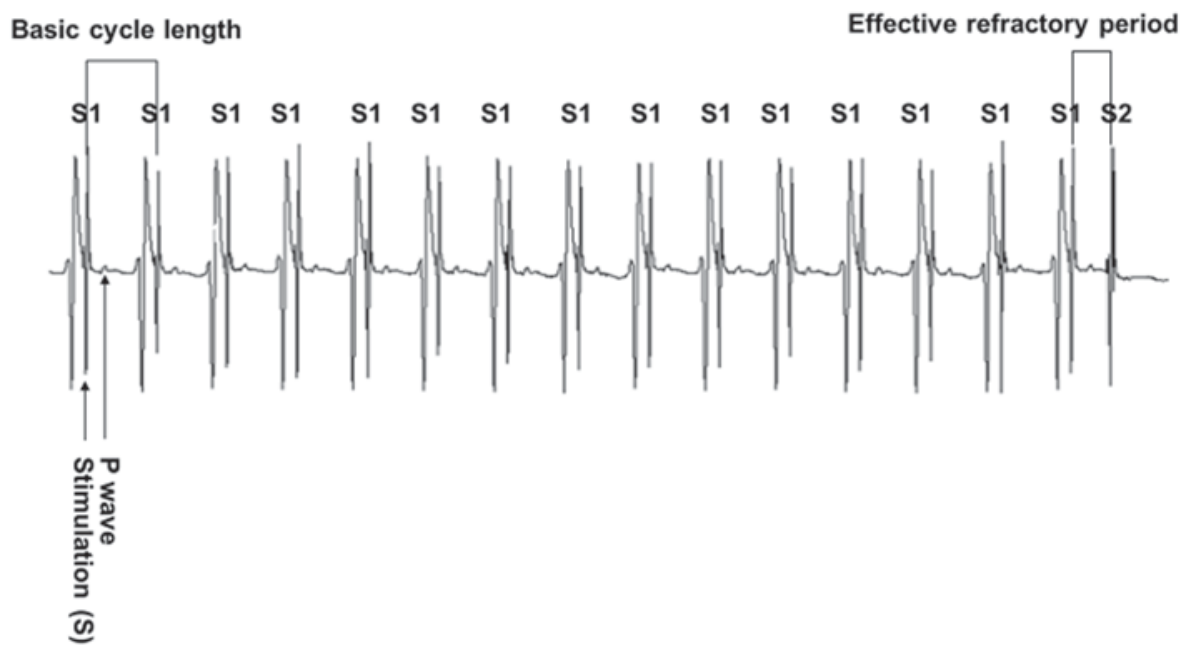

Figure 2. Measurement of the effective refractory period. The atrium was paced with a train of 15 S1s followed by a S2 with the coupling interval (S1-S2 interval) decreased in $5 \mathrm{msec}$ steps until capture no longer occurred. The longest S1-S2 interval failing to capture the atrium was regarded as the effective refractory period. S1, basic stimuli; S2, premature stimulus.

$150 \mathrm{msec}(\mathrm{P}<0.01)$, and from $55.2 \pm 1.6$ to $48.5 \pm 2.8 \mathrm{msec}$ at a $\mathrm{BCL}$ of $100 \mathrm{msec}(\mathrm{P}<0.05$; Fig. 3). Prior to pacing, a significant rate-dependent change in the ERP was observed $(\mathrm{P}<0.01$; Fig. 3). However, in the control group this was eliminated after pacing. Administration of EPA attenuated ERP shortening following pacing and maintained a significant difference in rate between a BCL of 100 and a $\mathrm{BCL}$ of $150 \mathrm{msec}(\mathrm{P}<0.05$; Fig. 3). A similar effect was observed with pitavastatin treatment $(\mathrm{P}<0.01$; Fig. 3). Furthermore, compared with the EPR before pacing, pitavastatin treatment increased the EPR after pacing; however, the mechanism remained unclear.

EPA treatment and the expression of cardiac ion channel genes. Short-term rapid atrial pacing significantly upregulated the mRNA levels of the $3 \mathrm{~K}^{+}$channel genes compared with the levels before pacing (Kcnd2, KChIP2 and Kcna5; $\mathrm{P}<0.05$; Fig. 4); however, no significant difference was observed in levels of Slc9a1 mRNA before and after pacing. EPA treatment suppressed the pacing-induced overexpression of Kcnd2, KChIP2 and Kcna5, and suppressed Slc9a1 expression to a level significantly lower than before pacing $(\mathrm{P}<0.01$; Fig. 4$)$. Treatment with pitavastatin attenuated the pacing-induced upregulation of Kcnd2 and KChIP2, and suppressed the expressions of Kcna5 and Slc9a1 to significantly below their pre-pacing values $(\mathrm{P}<0.05$; Fig. 4$)$.

Effect of EPA treatment on anticoagulant function and oxidative stress. In the control group, TM and TFPI gene expressions were significantly downregulated by rapid pacing $(\mathrm{P}<0.05$ and $\mathrm{P}<0.01$, respectively; Fig. 5). In the EPA treatment group, mRNA 
Table I. Primers used for polymerase chain reaction.

\begin{tabular}{lll}
\hline Gene & Direction & Sequence \\
\hline Kcna5 & Forward & 5-ATCACCAGCGGGTCCTCATAA-3 \\
Kcnd2 & Reverse & 5-GGAGGGTGTTGGGAAACTGC-3 \\
& Forward & 5-TCAGGACGCTCTGATAGTGCT-3 \\
KChIP2 & Reverse & 5-TCTGGGTATCGTTCCAGGGTG-3 \\
& Forward & 5-AGCGTGGAGGATGAGTTTGAAC-3 \\
Slc9a1 & Reverse & 5-TTCCCCGAAGAATCACTGACA-3 \\
TFPI & Forward & 5-TCATCCACCTCGGATCTTCCC-3 \\
& Reverse & 5-TCCTGAGAACAGGTAGCAGTC-3 \\
TM & Forward & 5-CAGGCGTCGGGTTACAAAAGA-3 \\
& Reverse & 5-GGAACTCAGAAAGCCTTGGTAAG-3 \\
eNOS & Forward & 5-AGTGTGCCAGTTCATAAGAATC-3 \\
Rac1 & Reverse & 5-GTTCTACAAAGGTTGGAAACTC-3 \\
& Forward & 5-GGCAAGACAGACTACACGAC-3 \\
GAPDH & Reverse & 5-ATCGCCGCAGACAAACAT-3 \\
& Forward & 5-ACGGAGCTGTTGGTAAAACCT-3 \\
\hline
\end{tabular}

Kcna5, potassium voltage-gated channel subfamily A member 5; Kcnd2, potassium voltage-gated channel subfamily D member 2; KChIP2, Kv channel-interacting protein 2; Slc9a1, solute carrier family 9 member A1; TFPI, tissue factor pathway inhibitor; TM, thrombomodulin; eNOS, endothelial nitric oxide synthase; Rac1, Ras-related C3 botulinum toxin substrate 1.

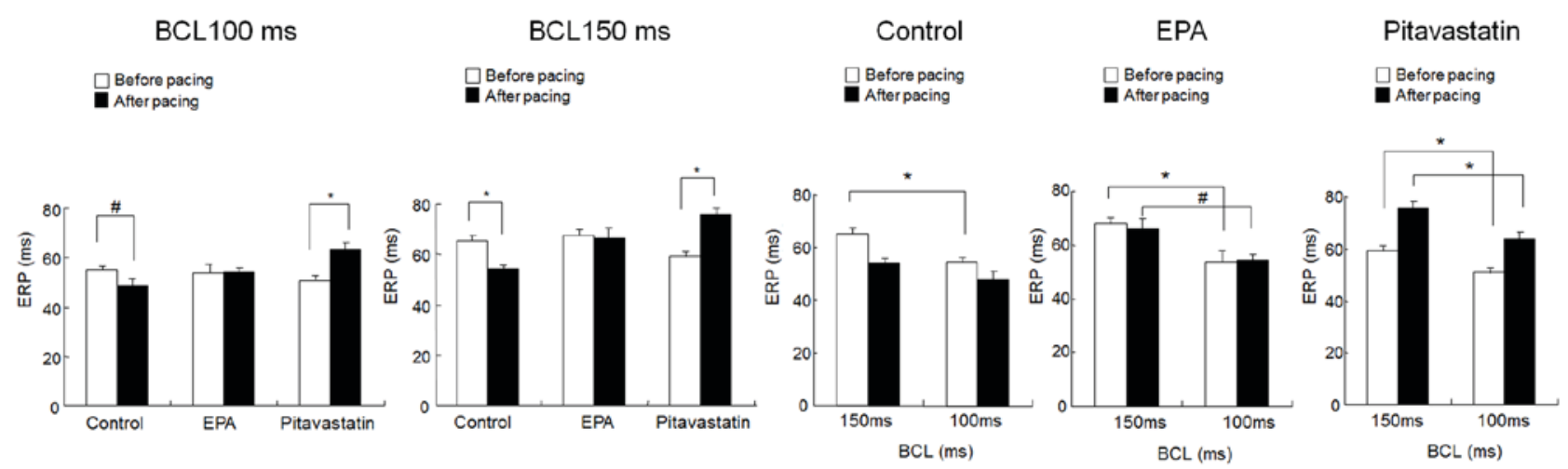

Figure 3. EPA pretreatment prevents the electrical remodeling of rapidly-paced atria. An 8-h pacing period significantly shortened the atrial ERP at BCLs of 100 and $150 \mathrm{msec}$ in the control group. Treatment with EPA or pitavastatin prevented these changes. Before pacing, ERPs significantly differed between BCLs of 100 and $150 \mathrm{msec}$. The 8-h rapid pacing period eleminated this difference in control mice, but had no influence on the mice in the EPA and pitavastatin groups. Data are presented as the mean + standard error of the mean. ${ }^{*} \mathrm{P}<0.05$ and ${ }^{*} \mathrm{P}<0.01$ as indicated. BCL, basic cycle length; EPA, eicosapentaenoic acid; ERP, effective refractory period.

expression levels of TFPI were not significantly affected by rapid pacing in the atrium; however, TM expression was significantly reduced after pacing compared with before $(\mathrm{P}<0.01$; Fig. 5). Pitavastatin treatment attenuated the pacing-induced downregulation of TM, with no significant differences observed between mRNA levels before and after pacing. Furthermore, TFPI expression in the pitavastatin group was significantly higher after compared with before pacing $(\mathrm{P}<0.05$; Fig. 5). In the control group, eNOS and Rac1 expression levels were significantly increased after pacing $(\mathrm{P}<0.01$ and $\mathrm{P}<0.05$, respectively; Fig. 5); however, EPA treatment attenuated this upregulation so that eNOS expression was not significantly different after pacing compared with before, and Racl levels were reduced to a level significantly lower than before pacing $(\mathrm{P}<0.01$; Fig. 5). Treatment with pitavastatin induced a significant decrease in the post-pacing levels of eNOS and Racl compared with before pacing $(\mathrm{P}<0.05$ and $\mathrm{P}<0.01$, respectively; Fig. 5).

\section{Discussion}

The results of the present study demonstrated that EPA and pitavastatin treatment were able to prevent electrical 


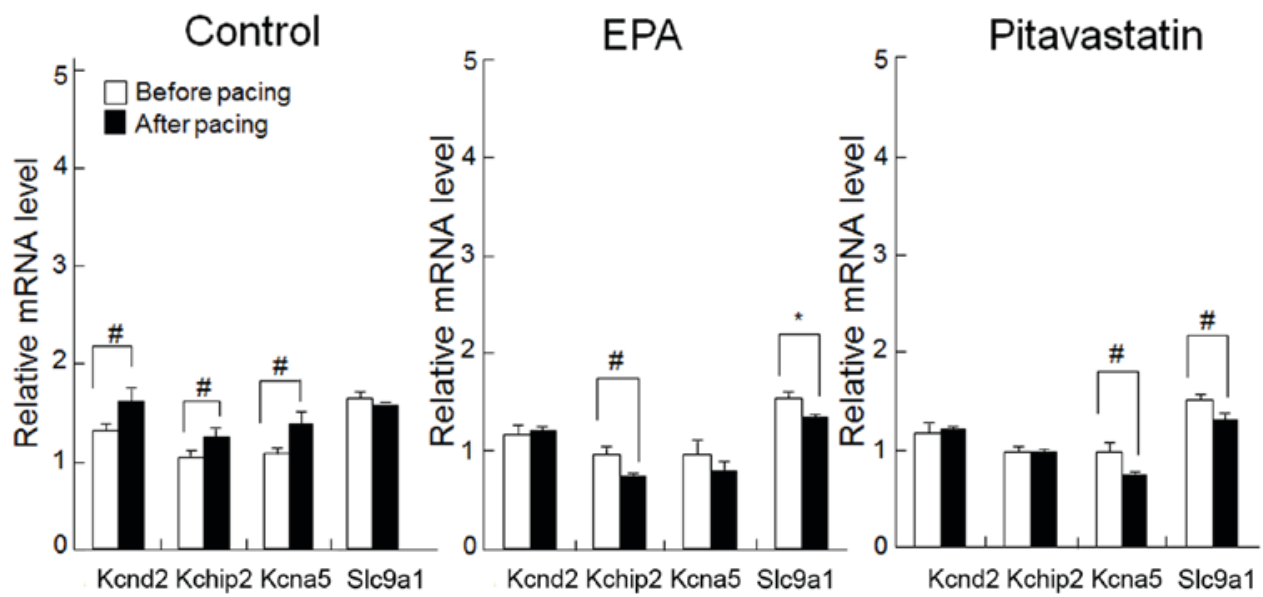

Figure 4. EPA pretreatment improves cardiac ion channel expression in rapidly-paced atria. The expression levels of the $\mathrm{K}^{+}$ion channel genes, Kcnd2, KChIP2 and Kcna5, increased after pacing in the control group and both EPA and pitavastatin pretreatment attenuated this effect. EPA and pitavastatin also suppressed the expression of Slc9a1; however, rapid pacing did not significantly influence its expression in the control group. Expression levels are relative to GAPDH Data are presented as the mean + standard error of the mean. ${ }^{~} \mathrm{P}<0.05$ and ${ }^{*} \mathrm{P}<0.01$ as indicated. EPA, eicosapentaenoic acid; Kcnd2, potassium voltage-gated channel subfamily D member 2; KChIP2, Kv channel-interacting protein 2; Kcna5, potassium voltage-gated channel subfamily A member 5; Slc9a1, solute carrier family 9 member A1.

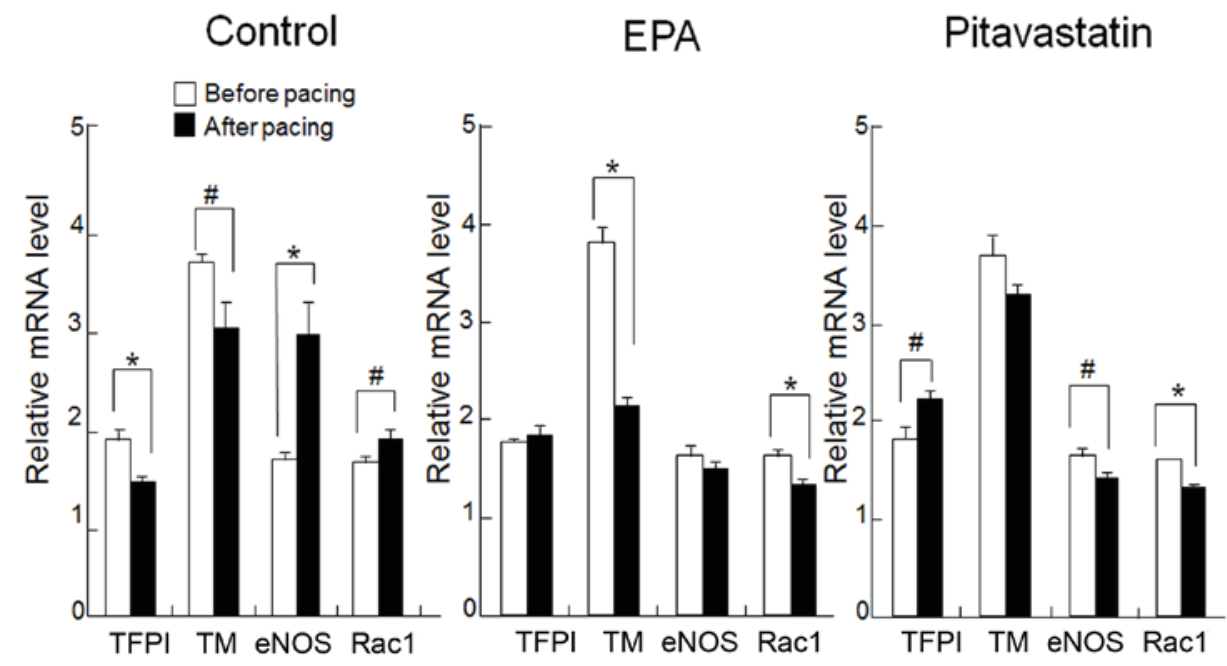

Figure 5. Effect of EPA pretreatment on the anticoagulant function and oxidative stress in rapidly-paced atria. The expression levels of intrinsic anticoagulant factors, TFPI and TM, were significantly suppressed following pacing in the control group, and Rac1 gene expression significantly increased compared with before pacing. Treatment with EPA or pitavastatin inhibited these changes. Expression levels are relative to GAPDH. Data are presented as the mean + standard error of the mean. "P $\mathrm{P}<0.05$ and ${ }^{\text {}} \mathrm{P}<0.01$ as indicated. EPA, eicosapentaenoic acid; TFPI, tissue factor pathway inhibitor; TM, thrombomodulin; eNOS, endothelial nitric oxide synthase; Rac1, Ras-related C3 botulinum toxin substrate 1.

remodeling in a murine model of rapid atrial pacing, prevent the modification of voltage-dependent $\mathrm{K}^{+}$channel gene expression and Slc9a1 expression, suppress atrial oxidative stress and attenuate the downregulation of the intrinsic anticoagulant factor, TFPI. Furthermore, pitavastatin treatment was demonstrated to ameliorate the pacing-induced downregulation of TM.

Dietary intake of polyunsaturated fatty acids has been reported to have beneficial effects on cardiovascular outcomes (24). A study by Mozaffarian et al (15) demonstrated that $\mathrm{n}-3$ fatty acids were associated with a lower incidence of AF development in a prospective, population-based cohort of
4,815 adults older aged $>65$ years. In a secondary prevention clinical trial with fish oil, it was reported that mortality associated with sudden cardiac events was reduced by $53 \%$ and total mortality was reduced by $41 \%$ following myocardial infarction in 11,323 patients treated with $1.0 \mathrm{~g} / \mathrm{day}$ of fish oil (25). Data from several experimental studies also reported the antiarrhythmic properties of n-3 fatty acids (26-29). It is well known that the effect of fish oil differs between forms (29-32). The incorporated $n-3$ polyunsaturated fatty acid has been reported to result in a significant prolongation of the atrial and pulmonary vein (PV) refractoriness and a decrease in the dispersion of the PV ERP (30-32). In contrast, 
free EPA and docosahexaenoic acid (DHA) may cause atrial conduction to slow significantly with a minimal effect on the tissue refractoriness (33). A study by Metcalf et al (34) reported that atrial accumulation of EPA and DHA was curvilinear over time and reached a maximum at 30 days. In the present study, multiple effects of a 6-week EPA/pitavastatin pretreatment were identified in a murine model of rapid atrial pacing.

The results of the present study suggested that a 6-week EPA pretreatment may prevent atrial electrical remodeling, similar to the effects of pitavastatin. In human hearts, the transient outward $\mathrm{K}^{+}$current, $\mathrm{I}_{\mathrm{to}}$, and the ultra-rapid delayed rectifier $\mathrm{K}^{+}$current, $\mathrm{I}_{\mathrm{Kur}}$, are important repolarization currents $(35,36)$. A study by Li et al (37) reported that EPA/DHA was able to inhibit the $I_{\text {to }}$ and $I_{K u r}$ in atrial cells isolated from patients with AF, which suggested an anti-AF effect of fish oil. In the present study, the expression levels of Kcna5, Kcnd2 and KChIP2 were significantly suppressed by EPA/pitavastatin pretreatment, which was consistent with the results of human studies. In addition, Jayachandran et al (7) reported that a sodium/hydrogen exchanger was important for the electrophysiological remodeling in a canine model of atrial pacing and a sodium/hydrogen exchange inhibitor may prevent such atrial remodeling. In the present study, the expression of the sodium/hydrogen exchanger gene, Slc9a1, was not influenced by atrial pacing. However, EPA or pitavastatin pretreatment suppressed Slc9a1 expression, which favored the recovery of the atrial remodeling.

The role of AF-induced oxidative stress in AF-related atrial remodeling has been observed extensively. Over the past two decades, studies have reported that ROS generated in the cardiovascular system are primarily derived from NOX, uncoupled eNOS, mitochondria and xanthine oxidase $(38,39)$. Rapid atrial pacing-induced refractoriness abbreviation and AF promotion are associated with an eNOS-dependent L-type $\mathrm{Ca}^{2+}$ current inhibition (40). Antioxidant vitamins were also reported to have beneficial effects in preventing atrial tachycardia remodeling (41). Furthermore, among the enzymatic systems, NOX has emerged as a major initiating source of increased ROS production in the cardiovascular system (42). As an important determinant of subunit assembly, the amount of the active Rac1 has been reported to be associated with NOX-dependent $\mathrm{O}_{2}$ production $(43,44)$. A study by Adam et al (45) reported that the left atria of patients with AF had a 4-fold increase in Racl total protein and membrane expression and an upregulation in Racl activity. In the present study, Racl and eNOS expressions were significantly increased in rapidly-paced murine atria, and EPA pretreatment effectively ameliorated this effect.

It is well known that decreased blood flow with a hypercoagulable state in the left atria of patients with AF increases the likelihood of clotting in the fibrillating atria $(46,47)$. In the present study, the effects of EPA pretreatment on two intrinsic anticoagulant factors, TM and TFPI, were investigated. The results indicated that the expression of these genes decreased significantly in the atrial-paced mice. EPA treatment caused the mRNA level of the TFPI to recover, whereas it had no effect on TM. However, pitavastatin treatment improved both gene expressions.

At present, therapy for patients with AF is predominantly palliative. Ideally, research aims to identify an effective treatment or a preventive strategy for AF that will prevent triggers, substrates, or both, from occurring. As a promising upstream therapy for AF, the effect of fish oil/pitavastatin has been intensively observed. Although various studies have demonstrated the beneficial effects of these treatments, translating these findings into human use has had mixed results due to inconsistencies in data in both clinical and experimental studies. AF is a complex disease and at present is not well understood. Further studies on the mechanism of fish oil/pitavastatin may lead to a better understanding of the underlying mechanisms of AF and provide more potential therapies.

In conclusion, the results of the present study demonstrated that pretreatment with EPA or pitavastatin had pleiotropic effects on rapid pacing-induced atrial remodeling, including suppression of electrical remodeling and the expression of corresponding ion channel genes, reducing oxidative stress and improving anticoagulant activity. Furthermore, additional extensive studies on the detailed molecular pathways associated with these beneficial effects may provide greater understanding of atrial fibrillation and allow for the development of novel treatments.

\section{Acknowledgements}

The present study was supported by the Natural Science Foundation of Ningbo City (grant no. 2014A610269), the Zhejiang Provincial Medical and Health Technology Program (grant no. 2016KYB262) and the Zhejiang Provincial Natural Science Foundation (grant nos. LY12H02001 and LY14H090003).

\section{References}

1. Wann LS, Curtis AB, Ellenbogen KA, Estes NA III, Ezekowitz MD, Jackman WM, January CT, Lowe JE, Page RL, Slotwiner DJ, et al: 2011 ACCF/AHA/HRS focused update on the management of patients with atrial fibrillation (update on dabigatran): A report of the American College of cardiology Foundation/American heart association task force on practice guidelines. J Am Coll Cardiol 57: 1330-1337, 2011

2. Benjamin EJ, Wolf PA, D'Agostino RB, Silbershatz H, Kannel WB and Levy D: Impact of atrial fibrillation on the risk of death: The Framingham Heart Study. Circulation 98: 946-952, 1998.

3. Nattel S, Burstein B and Dobrev D: Atrial remodeling and atrial fibrillation: Mechanisms and implications. Circ Arrhythm Electrophysiol 1: 62-73, 2008.

4. Kourliouros A, Savelieva I, Kiotsekoglou A, Jahangiri M and Camm J: Current concepts in the pathogenesis of atrial fibrillation. Am Heart J 157: 243-252, 2009.

5. Allessie M, Ausma J and Schotten U: Electrical, contractile and structural remodeling during atrial fibrillation. Cardiovasc Res 54: 230-246, 2002.

6. Yamashita T, Murakawa Y, Hayami N, Fukui Ei, Kasaoka Y, Inoue $\mathrm{M}$ and Omata $\mathrm{M}$ : Short-term effects of rapid pacing on mRNA level of voltage-dependent $\mathrm{K}(+)$ channels in rat atrium: Electrical remodeling in paroxysmal atrial tachycardia. Circulation 101: 2007-2014, 2000.

7. Jayachandran JV, Zipes DP, Weksler J and Olgin JE: Role of the $\mathrm{Na}(+) / \mathrm{H}(+)$ exchanger in short-term atrial electrophysiological remodeling. Circulation 101: 1861-1866, 2000.

8. Korantzopoulos P, Kolettis TM, Galaris D and Goudevenos JA: The role of oxidative stress in the pathogenesis and perpetuation of atrial fibrillation. Int J Cardiol 115: 135-143, 2007.

9. Sarfstein R, Gorzalczany Y, Mizrahi A, Berdichevsky Y, Molshanski-Mor S, Weinbaum C, Hirshberg M, Dagher MC and Pick E: Dual role of Rac in the assembly of NADPH oxidase, tethering to the membrane and activation of p67phox: A study based on mutagenesis of p67phox-Rac1 chimeras. J Biol Chem 279: 16007-16016, 2004. 
10. Adam O, Frost G, Custodis F, Sussman MA, Schäfers HJ, Böhm M and Laufs U: Role of Rac1 GTPase activation in atrial fibrillation. J Am Coll Cardiol 50: 359-367, 2007.

11. Wolf PA, Abbott RD and Kannel WB: Atrial fibrillation as an independent risk factor for stroke: The Framingham Study. Stroke 22: 983-988, 1991.

12. Yamashita T, Sekiguchi A, Iwasaki YK, Sagara K, Hatano S, Iinuma $\mathrm{H}$, Aizawa $\mathrm{T}$ and $\mathrm{Fu}$ LT: Thrombomodulin and tissue factor pathway inhibitor in endocardium of rapidly paced rat atria. Circulation 108: 2450-2452, 2003.

13. Miyasaka Y, Barnes ME, Gersh BJ, Cha SS, Bailey KR, Abhayaratna WP, Seward JB and Tsang TS: Secular trends in incidence of atrial fibrillation in Olmsted County, Minnesota, 1980 to 2000, and implications on the projections for future prevalence. Circulation 114: 119-125, 2006.

14. Savelieva I, Kourliouros A and Camm J: Primary and secondary prevention of atrial fibrillation with statins and polyunsaturated fatty acids: Review of evidence and clinical relevance. Naunyn Schmiedebergs Arch Pharmacol 381: 1-13, 2010.

15. Mozaffarian D, Psaty BM, Rimm EB, Lemaitre RN, Burke GL, Lyles MF, Lefkowitz D and Siscovick DS: Fish intake and risk of incident atrial fibrillation. Circulation 110: 368-373, 2004.

16. Brouwer IA, Heeringa J, Geleijnse JM, Zock PL and Witteman JC: Intake of very long-chain n-3 fatty acids from fish and incidence of atrial fibrillation. The Rotterdam Study. Am Heart J 151: 857-862, 2006.

17. Frost L and Vestergaard P: n-3 Fatty acids consumed from fish and risk of atrial fibrillation or flutter: The danish diet, cancer, and health study. Am J Clin Nutr 81: 50-54, 2005.

18. Daviglus ML, Stamler J, Orencia AJ, Dyer AR, Liu K, Greenland P, Walsh MK, Morris D and Shekelle RB: Fish consumption and the 30 -year risk of fatal myocardial infarction. N Engl J Med 336: 1046-1053, 1997.

19. Hopper L, Ness A, Higgins JP, Moore T and Ebrahim S: GISSI-Prevenzione trial. Lancet 354: 1557, 1999.

20. Yokoyama M, Origasa H, Matsuzaki M, Matsuzawa Y, Saito Y, Ishikawa Y, Oikawa S, Sasaki J, Hishida H, Itakura $\mathrm{H}$, et al: Effects of eicosapentaenoic acid on major coronary events in hypercholesterolaemic patients (JELIS): A randomised open-label, blinded endpoint analysis. Lancet 369: 1090-1098, 2007.

21. Laurent G, Moe G, Hu X, Holub B, Leong-Poi H, Trogadis J, Connelly K, Courtman D, Strauss BH and Dorian P: Long chain n-3 polyunsaturated fatty acids reduce atrial vulnerability in a novel canine pacing model. Cardiovasc Res 77: 89-97, 2008.

22. Sarrazin JF, Comeau G, Daleau P, Kingma J, Plante I, Fournier D and Molin F: Reduced incidence of vagally induced atrial fibrillation and expression levels of connexins by $n-3$ polyunsaturated fatty acids in dogs. J Am Coll Cardiol 50: 1505-1512, 2007.

23. Tong M, Watanabe E, Yamamoto N, Nagahata-Ishiguro M, Maemura K, Takeda N, Nagai R and Ozaki Y: Circadian expressions of cardiac ion channel genes in mouse might be associated with the central clock in the SCN but not the peripheral clock in the heart. Biol Rhythm Res 44: 519-530, 2013.

24. Hu FB, Manson JE and Willett WC: Types of dietary fat and risk of coronary heart disease: A critical review. J Am Coll Nutr 20: $5-19,2001$.

25. Marchioli R, Barzi F, Bomba E, Chieffo C, Di Gregorio D, Di Mascio R, Franzosi MG, Geraci E, Levantesi G, Maggioni AP, et al: Early protection against sudden death by omega-3 polyunsaturated fatty acids after myocardial infarction: Time-course analysis of the results of the Gruppo Italiano per lo Studio della Sopravvivenza nell'Infarto Miocardico (GISSI)-Prevenzione. Circulation 105: 1897-1903, 2002

26. Leaf A, Kang JX, Xiao YF and Billman GE: Clinical prevention of sudden cardiac death by n-3 polyunsaturated fatty acids and mechanism of prevention of arrhythmias by $n-3$ fish oils Circulation 107: 2646-2652, 2003

27. Leaf A, Xiao YF, Kang JX and Billman GE: Prevention of sudden cardiac death by $n-3$ polyunsaturated fatty acids. Pharmacol Ther 98: 355-377, 2003.

28. Jones PJ and Lau VW: Effect of n-3 polyunsaturated fatty acids on risk reduction of sudden death. Nutr Rev 60: 407-409, 2002.
29. Lee KW and Lip GY: The role of omega-3 fatty acids in the secondary prevention of cardiovascular disease. QJM 96: 465-480, 2003

30. Kumar S, Sutherland F, Rosso R, Teh AW, Lee G, Heck PM, Feldman A, Medi C, Watt S, Garg ML and Sparks PB: Effects of chronic omega-3 polyunsaturated fatty acid supplementation on human atrial electrophysiology. Heart Rhythm 8: 562-568, 2011.

31. Kumar S, Sutherland F, The AW, Heck PM, Lee G, Garg ML and Sparks PB: Effects of chronic omega-3 polyunsaturated fatty acid supplementation on human pulmonary vein and left atrial electrophysiology in paroxysmal atrial fibrillation. Am J Cardiol 108: 531-535, 2011.

32. Ninio DM, Murphy KJ, Howe PR and Saint DA: Dietary fish oil protects against stretch-induced vulnerability to atrial fibrillation in a rabbit model. J Cardiovasc Electrophysiol 16: 1189-1194, 2005.

33. Kumar S, Sutherland F, Lee JM, Robinson T, Heck PM, Wong MC, Kelland NF, Garg ML and Sparks PB: Effects of high dose intravenous fish oil on human atrial electrophysiology: Implications for possible anti- and pro-arrhythmic mechanisms in atrial fibrillation. Int J Cardiol 168: 2754-2760, 2013.

34. Metcalf RG, James MJ, Gibson RA, Edwards JR, Stubberfield J, Stuklis R, Roberts-Thomson K, Young GD and Cleland LG: Effects of fish-oil supplementation on myocardial fatty acids in humans. Am J Clin Nutr 85: 1222-1228, 2007.

35. Li GR, Feng J, Wang Z, Fermini B and Nattel S: Comparative mechanisms of 4-aminopyridine-resistant Ito in human and rabbit atrial myocytes. Am J Physiol 269: H463-H472, 1995.

36. Li GR, Feng J, Yue L and Carrier M: Transmural heterogeneity of action potentials and Ito1 in myocytes isolated from the human right ventricle. Am J Physiol 275: H369-H377, 1998.

37. Li GR, Sun HY, Zhang XH, Cheng LC, Chiu SW, Tse HF and Lau CP: Omega-3 polyunsaturated fatty acids inhibit transient outward and ultra-rapid delayed rectifier $\mathrm{K}+$ currents and $\mathrm{Na}+$ current in human atrial myocytes. Cardiovasc Res 81: 286-293, 2009.

38. Cai H: Hydrogen peroxide regulation of endothelial function: Origins, mechanisms, and consequences. Cardiovasc Res 68: 26-36, 2005.

39. Cai H, Griendling KK and Harrison DG: The vascular NAD(P)H oxidases as therapeutic targets in cardiovascular diseases. Trends Pharmacol Sci 24: 471-478, 2003.

40. Shiroshita-Takeshita A, Brundel BJ, Lavoie J and Nattel S: Prednisone prevents atrial fibrillation promotion by atrial tachycardia remodeling in dogs. Cardiovasc Res 69: 865-875, 2006.

41. Youn JY, Zhang J, Zhang Y, Chen H, Liu D, Ping P, Weiss J and Cai H: Oxidative stress in atrial fibrillation: an emerging role of NADPH oxidase. J Mol Cell Cardiol 62: 72-79, 2013.

42. Carnes CA, Chung MK, Nakayama T, Nakayama H, Baliga RS, Piao S, Kanderian A, Pavia S, Hamlin RL, McCarthy PM, et al: Ascorbate attenuates atrial pacing-induced peroxynitrite formation and electrical remodeling and decreases the incidence of postoperative atrial fibrillation. Circ Res 89: E32-E38, 2001.

43. Maack C, Kartes T, Kilter H, Schafers HJ, Nickenig G, Böhm M and Laufs U: Oxygen free radical release in human failing myocardium is associated with increased activity of rac1-GTPase and represents a target for statin treatment. Circulation 108: 1567-1574, 2003.

44. Price MO, Atkinson SJ, Knaus UG and Dinauer MC: Rac activation induces NADPH oxidase activity in transgenic COSphox cells, and the level of superoxide production is exchange factor-dependent. J Biol Chem 277: 19220-19228, 2002.

45. Adam O, Frost G, Custodis F, Sussman MA, Schäfers HJ, Böhm M and Laufs U: Role of Rac1 GTPase activation in atrial fibrillation. J Am Coll Cardiol 50: 359-367, 2007.

46. Gustafsson C, Blombäck M, Britton M, Hamsten A and Svensson J: Coagulation factors and the increased risk of stroke in nonvalvular atrial fibrillation. Stroke 21: 47-51, 1990.

47. Fatkin D, Kelly RP and Feneley MP: Relations between left atrial appendage blood flow velocity, spontaneous echocardiographic contrast and thromboembolic risk in vivo. J Am Coll Cardiol 23: 961-969, 1994. 